\title{
Fourier multipliers on the real Hardy spaces
}

\author{
Sebastian Król
}

\begin{abstract}
We provide a variant of Hytönen's embedding theorem, which allows us to extend and unify several sufficient conditions for a function to be a Fourier multiplier on the real Hardy spaces.
\end{abstract}

Mathematics Subject Classification. 42B15.

Keywords. Fourier multiplier, Weighted Hardy space, Fourier embedding.

1. Introduction. Multipliers on the real Hardy spaces $H^{p}\left(\mathbb{R}^{n}\right)(p \in(0, \infty))$ have attracted much attention in the literature. The note continues lines of research due to Hytönen [5] and Kolomoitsev [6,7] extending earlier pioneering results, in particular, by Hörmander [4], Calderón and Torchinsky [2], Kurtz and Wheeden [8], De Michel and Inglis [3], Miyachi [9,10], Baerstein and Sawyer [1], Stömberg and Torchinsky [12].

The obtained multiplier conditions are two-fold. This corresponds to two different approaches of measuring the smoothness of multiplier functions which we apply in the paper. The diverse character is well illustrated by considering the minimal number of derivatives for checking either of the conditions.

More precisely, as a consequence of Theorem 4.1 which is obtained by adaptation of Hytönen's $\infty$-norm approach from [5], we get the following sufficient condition for a function to be a Fourier multiplier on the real Hardy spaces $H^{p}\left(\mathbb{R}^{n}\right)(n \in \mathbb{N})$.

We write $\chi_{S_{\mu}}$ for the characteristic function of the set $S_{\mu}:=\left\{x \in \mathbb{R}^{n}\right.$ : $\left.2^{\mu-1}<|x|<2^{\mu+1}\right\}$ and set

$$
\mathcal{I}_{l, d}:=\left\{\alpha \in \mathbb{N}_{0}^{n}:|\alpha|_{\infty} \leq d \text { and }|\alpha|_{1} \leq l\right\} \quad(d, l \in \mathbb{N}) .
$$

S. Król was partially supported by the Alexander von Humboldt Foundation and Narodowe Centrum Nauki Grant DEC-2011/03/B/ST1/00407. 
Theorem 1.1. Let $d \in \mathbb{N}, t \in(1,2]$, and $l \in\{\lfloor n / t\rfloor+1, \ldots, d n\}$. Let $m \in$ $L^{\infty}\left(\mathbb{R}^{n}\right)$ with $D^{\alpha} m \in L_{\text {loc }}^{1}\left(\mathbb{R}^{n}\right)$ for all multi-indices $\alpha \in \mathcal{I}_{l, d}$. Suppose that there exists a constant $\rho>0$ such that

$$
\sup _{\mu \in \mathbb{Z}, \alpha \in \mathcal{I}_{l, d}}\left\|\chi_{S_{\mu}} m\right\|_{\infty}^{\rho}\left\|2^{\mu\left(|\alpha|_{1}-n / t\right)} \chi_{S_{\mu}} D^{\alpha} m\right\|_{t}<\infty .
$$

Then $m$ is a Fourier multiplier on $H^{p}\left(\mathbb{R}^{n}\right)$ for all $p \in(0,2]$ such that

$$
\frac{1}{p}-\frac{1}{2}<\frac{1}{1+\rho}\left(\frac{l}{n^{2}}+\frac{1}{2}-\frac{1}{t n}\right) .
$$

In the case of $\liminf |\mu| \rightarrow \infty\left\|\chi_{S_{\mu}} m\right\|_{\infty}>0$, the condition (1) is equivalent to the Hytönen-type condition, i.e. $\sup _{\mu \in \mathbb{Z}, \alpha \in \mathcal{I}_{l, d}}\left\|2^{\mu\left(|\alpha|_{1}-n / t\right)} \chi_{S_{\mu}} D^{\alpha} m\right\|_{t}<\infty$, studied in [5]; see also Remark 4.3.

Otherwise, it presents a diverse feature. For instance, if $|m(x)| \leq C(1+$ $|x|)^{-a}\left(x \in \mathbb{R}^{n}\right)$ for some $a>0$ and

$$
\sup _{\mu \in \mathbb{Z}, \alpha \in \mathcal{I}_{l, d}} \min \left(2^{-\mu b}, 1\right)\left\|2^{\mu\left(|\alpha|_{1}-n / t\right)} \chi_{S_{\mu}} D^{\alpha} m\right\|_{t}<\infty
$$

for some $b \geq 0$, then the conclusion of Theorem 1.1 holds with $\rho=b / a$. This shows that under an additional information on the decay of a function $m$, a weakened Hytönen-type condition still ensures that $m$ is a Fourier multiplier on $H^{p}\left(\mathbb{R}^{n}\right)$ for some $p<2$.

The extension of the classical Hörmander-type condition which involves an additional analytic behaviour of the multiplier function goes back to $[3,9,10]$. More recently, such conditions were systematically studied by Kolomoitsev $[6,7]$.

The Kolomoitsev-type conditions differ from (1) in a few points. For the convenience of the reader we restate $[6$, Theorem 2$]$ and a special case of $[6$, Theorem 1], which can be reformulated in our setting as follows. Set

$$
\mathcal{I}_{d}:=\left\{j e_{i}: i=1, \ldots, n, j=0,1, \ldots, d\right\},
$$

where $\left\{e_{i}\right\}_{i=1}^{n}$ is the canonical basis of $\mathbb{R}^{n}$.

Theorem A (Kolomoitsev, [6, Theorems 1 and 2]). Let $d>n / 2$ and let $m \in$ $L^{\infty}\left(\mathbb{R}^{n}\right)$ with $D^{\alpha} m \in L_{\text {loc }}^{1}\left(\mathbb{R}^{n}\right)$ for all $\alpha \in \mathcal{I}_{d}$. Suppose that there exists a constant $\rho>0$ such that

$$
\sup _{\mu \in \mathbb{Z}, \alpha \in \mathcal{I}_{d}}\left\|\chi_{S_{\mu}} m\right\|_{\infty}^{\rho}\left\|2^{\mu\left(|\alpha|_{1}-n / 2\right)} \chi_{S_{\mu}} D^{\alpha} m\right\|_{2}<\infty .
$$

Then $m$ is a Fourier multiplier on $H^{p}\left(\mathbb{R}^{n}\right)$ for every $p \in(0,2]$ such that

$$
\frac{1}{p}-\frac{1}{2} \leq \frac{1}{1+\rho} \frac{d}{n}
$$

Note that the sets of multi-indices $\alpha$ involved in the conditions (1) and (4) differ essentially, as well as the corresponding conclusions. A close analysis shows that neither result is superior in general and, as soon as $n \geq 2$, the one or the other might be better suited to a particular situation. 
For instance, note that in order to use Theorem A to verify whether bounded functions $m$, which are locally in the Sobolev spaces $W_{2}^{d}\left(\mathbb{R}^{n}\right)$, are Fourier multipliers on any desired $H^{p}\left(\mathbb{R}^{n}\right)$ for $p \in(0,1)$, one needs that the smoothness parameter $d$ is grater than $n / 2$. Theorem 1.1 shows that this restriction on the parameter $d$ can be relaxed, if we have an additional information on the behaviour of the mixed partial derivatives $D^{\alpha} m, \alpha \in \mathcal{I}_{d, l}$, with $l>n / 2$. Note that functions which are not locally in $W_{2}^{\lfloor n / 2\rfloor+1}\left(\mathbb{R}^{n}\right)$ but satisfy the condition (1) for $d \leq n / 2, l \in\{\lfloor n / 2\rfloor+1, \ldots, d n\}$ and $\rho>0$ small, can be easily constructed.

On the other hand, if (1) holds for $d>n / 2, l=d$, and $t=2$, then we get that the right-hand side of (2) is strictly less than that of (5). Therefore, Theorem 1.1 does not reproduce Theorem A. It should be also underlined that Kolomoitsev's result is sharp; see [6, Remark 1, p. 1565]. We do not know if one can modify Hytönen's $\infty$-approach, which is involved in the proof of Theorem 4.1, to remove this drawback and cover Theorem A.

We point out that the Fourier embedding result which is applied (implicitly) in the proofs of Kolomoitsev's multiplier theorems says that the Fourier transform maps the Besov space $B_{t, q}^{s}\left(\mathbb{R}^{n}\right)$ isomorphically onto $K_{t^{\prime}, q}^{s}\left(\mathbb{R}^{n}\right)$ for every $s \geq 0,0<q \leq \infty$, and $t=2$, where

$$
K_{t^{\prime}, q}^{s}\left(\mathbb{R}^{n}\right):=\left\{f \in L^{t^{\prime}}\left(\mathbb{R}^{n}\right): \sum_{\mu \in \mathbb{Z}} 2^{\mu s q}\left(\int_{S_{\mu}}|f|^{t^{\prime}}\right)^{q / t^{\prime}}<\infty\right\}
$$

see Baernstein and Sawyer [1, (2.2) p. 12]. At a first glance, this embedding result differs from Hytönen's one, [5, Propositions 3.1 and 3.4], which is involved in the proof of Theorem 1.1. However, in Section 3 we show that the difference can be made, in a sense, only of geometrical character which is relevant to divisions of $\mathbb{R}^{n}$ corresponding to the sets of multi-indices $\mathcal{I}_{d}$ and $\mathcal{I}_{d, l}$.

More precisely, we give a natural modification of [5, Propositions 3.1 and 3.4], see Lemma 3.1, which together with a characterisation of Besov spaces, see (9), shows immediately that $B_{t, q}^{s}\left(\mathbb{R}^{n}\right)$ embedds in $K_{t^{\prime}, q}^{s}\left(\mathbb{R}^{n}\right)$ for every $s \geq 0$, $t \in[1,2]$, and $t^{\prime} \leq q \leq \infty$. It allows us to extend and unify some results known in the literature.

In particular, combining Lemma 3.1 with techniques from [5], we extend the Strömberg-Torchinsky results, [12, Theorems 5 and 6, Chapter XI], which fall outside the scope of the treatment in [5]; see e.g. [5, Example 9.7]. We refer the reader to Theorems 3.4 and 3.7 for more details.

We conclude with some additional comments. Except for some details, we reproduce ideas which have been presented in [5] and the standard techniques from the multiplier theory developed in the above cited papers. Therefore, the presentation of the proofs of our results is restricted to providing only main supplementary observations which should be made. It requires an adaptation of the techniques developed in the corresponding papers. This allows us to keep the novelty of the paper in a more transparent way.

Moreover, the presentation is restricted to the scalar-valued case. The vector-valued counterparts of our results, with having a priori boundedness 
on one $L^{p}$ space of vector-valued functions, can be easily obtained by standard arguments. The interested reader can for instance mimic the presentation given in [5].

2. Preliminaries. We follow the notation introduced in [5]. For an arbitrary function $f: \mathbb{R}^{n} \rightarrow \mathbb{C}$, we define the translation $\tau_{h}$ and the difference operators $\Delta_{h}^{d}$ and $\partial_{h}^{\alpha}$ as follows:

$$
\begin{gathered}
\tau_{h} f(x):=f(x-h) \quad\left(x \in \mathbb{R}^{n}\right), \quad \Delta_{h} f:=f-\tau_{h} f, \\
\partial_{h}^{\alpha}:=\Pi_{i=1}^{n} \Delta_{h_{i} e_{i}}^{\alpha_{i}}=\sum_{\beta \leq \alpha}\left(\begin{array}{c}
\alpha \\
\beta
\end{array}\right)(-1)^{|\beta|} \tau_{\beta h} \quad\left(h \in \mathbb{R}^{n}, \alpha \in \mathbb{N}_{0}\right),
\end{gathered}
$$

where $e_{i}$ stands for the $i$ th standard unit vector in $\mathbb{R}^{n}, h \beta:=\left(h_{i} \beta_{i}\right)_{i=1}^{n}$, and by $\Delta_{h}^{j}\left(j \in \mathbb{N}_{0}\right)$ we mean the $j$-fold iteration of $\Delta_{h}$ with the convention that $\Delta_{h}^{0}$ is the identity. For a multi-index $\alpha:=\left(\alpha_{i}\right) \in \mathbb{N}_{0}^{n}$, we set $|\alpha|=|\alpha|_{1}=\sum_{i=1}^{n} \alpha_{i}$ and $|\alpha|_{\infty}:=\max _{i=1, \ldots, n} \alpha_{i}$ to denote its 1-norm and $\infty$-norm, respectively.

Throughout, $m$ denotes a bounded, measurable, complex-valued function on $\mathbb{R}^{n}$. Let $\psi \in \mathcal{C}^{\infty}\left(\mathbb{R}^{n}\right)$ with the range $[0,1]$, supp $\psi \subset \overline{B(0,1)}$, and $\psi \equiv 1$ on $\overline{B\left(0, \frac{1}{2}\right)}$. Let $\phi:=\psi-\psi(2 \cdot)$ and $\phi_{\mu}:=\phi\left(2^{-\mu}\right), \mu \in \mathbb{Z}$, be a homogeneous resolution of unity. Moreover, let $m_{\mu}:=m \phi_{\mu}(\mu \in \mathbb{Z})$ denote the dyadic parts

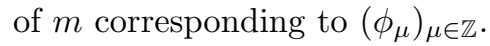

To illustrate our general results expressed in the terms of the difference operators we restate relevant Hörmander-type conditions studied by Strömberg and Torchinsky in [12, Chapter XI].

For real numbers $l \geq 0$ and $t \in[1,2]$ we say that $m$ satisfies the condition $M(t, l)$, and write $m \in M(t, l)$ if

$$
\left(\int_{R \leq|x|<2 R}\left|D^{\alpha} m(\xi)\right|^{t} \mathrm{~d} \xi\right)^{1 / t} \leq C R^{n / t-|\alpha|} \quad(R>0)
$$

for all multi-indices $\alpha$ with $|\alpha| \leq l$ when $l$ is a positive integer, and when $l$ is not an integer, in addition,

$\left(\int_{R<|\xi|<2 R}\left|D^{\alpha} m(\xi)-D^{\alpha} m(\xi-\zeta)\right|^{t} \mathrm{~d} \xi\right)^{1 / t} \leq C R^{n / t-|\alpha|}(|\zeta| / R)^{\epsilon} \quad(2|\zeta|<R)$

for all multi-indices $\alpha$ with $|\alpha|=\lfloor l\rfloor$, where $\epsilon:=l-\lfloor l\rfloor$.

In [12], Strömberg and Torchinsky considered how the behaviour of the Fourier transform of $m$ reflects the fact that $m \in M(t, l)$. They proved, in particular, that $k:=\widehat{m} \in K\left(t^{\prime}, l-n / t\right)$ if $l>n / t$; see [12, Lemma 1 , Chapter $\mathrm{XI}]$. Here, for $s \in[1, \infty]$ and $r>0$, we write $k \in K(s, r)$ if:

$$
\left(\int_{R \leq|x|<2 R}\left|D^{\alpha} k(x)\right|^{s} \mathrm{~d} x\right)^{1 / s} \leq C R^{n / s^{\prime}-n-|\alpha|} \quad(R>0)
$$




$$
\begin{aligned}
& \left(\int_{R<|\xi|<2 R}\left|D^{\beta} k(x)-D^{\beta} k(x-z)\right|^{s} \mathrm{~d} x\right)^{1 / s} \\
& \quad \leq C R^{n / s^{\prime}-|\alpha|} \sigma\left(\frac{|z|}{R} ; r-\lfloor r\rfloor^{-}\right) \quad(2|z|<R)
\end{aligned}
$$

for all multi-indices $\alpha$ and $\beta$ with $|\alpha| \leq\lfloor r\rfloor^{-}$and $|\beta|=\lfloor r\rfloor^{-}$, where $\lfloor r\rfloor^{-}$ denotes the largest integer strictly less than $r$, and $\sigma(h ; \epsilon):=h^{\epsilon}$ if $\epsilon \in(0,1)$, and $\sigma(h ; 1):=-h \log h$ if $\epsilon=1(h \in(0,1))$.

Subsequently, if $k_{\mu}:=k * \phi_{\mu}(\mu \in \mathbb{Z})$, and the functions $\sum_{-\nu}^{\nu} k_{\mu}, \nu \in \mathbb{N}$, satisfy the condition $K(s, r)$ uniformly with respect to $\nu \in \mathbb{N}$, then we also write $k \in K(s, r)$.

Following [11], given $1 \leq r \leq \infty$, we say that a function $k \in L_{l o c}^{1}\left(\mathbb{R}^{n} \backslash\{0\}\right)$ satisfies the $\left(D_{r}\right)$-condition, and write $k \in\left(D_{r}\right)$, if there exists a sequence $\left(c_{j}\right) \in l^{1}$ such that

$$
\left(\int_{2^{j}|y|<|x| \leq 2^{j+1}|y|}|k(x-y)-k(x)|^{r} \mathrm{~d} x\right)^{1 / r} \leq c_{j} 2^{-j n / r^{\prime}}|y|^{-n / r^{\prime}} \quad\left(j \in \mathbb{N}, y \in \mathbb{R}^{n}\right)
$$

(for $r=\infty$ this condition is understood in the usual way). Note that $\left(D_{1}\right)$ is the classical Hörmander condition. Moreover, for $k:=\widehat{m}$ with $m \in L^{\infty}$ we also write $k \in\left(D_{r}\right)$ if $\sum_{\mu=-\nu}^{\nu} k_{\mu} \in\left(D_{r}\right)$ uniformly in $\nu \in \mathbb{N}$.

We refer the reader to [12] for the background on weighted Hardy spaces $H_{w}^{p}\left(\mathbb{R}^{n}\right)$, and relevant classes of weights, i.e. the class of weights satisfying the Muckenhoupt $A_{s}$-condition, the reverse Hölder $R H_{r}$-condition, and the doubling $D_{b}$-condition; see $[12$, Chapter I].

We say that a function $m \in L^{\infty}\left(\mathbb{R}^{n}\right)$ is a Fourier multiplier on $H_{w}^{p}\left(\mathbb{R}^{n}\right)$, and write $m \in \mathcal{M}\left(H_{w}^{p}\right)$ if the operator $T$ given by

$$
(T f)^{\wedge}=m \widehat{f} \quad\left(f \in \widehat{\mathcal{D}}_{0}\right)
$$

extends to a bounded operator on $H_{w}^{p}\left(\mathbb{R}^{n}\right)$, where $\widehat{D}_{0}$ stands for $\left\{f \in S\left(\mathbb{R}^{n}\right)\right.$ : $\operatorname{supp} \hat{f}$ is compact and $0 \notin \operatorname{supp} \hat{f}\}$.

Finally, recall that $H_{w}^{p}\left(\mathbb{R}^{n}\right)$ is identical to $L_{w}^{p}\left(\mathbb{R}^{n}\right)$ for every $p \geq s$ and $w \in A_{s}$, where $s>1$; see $[12$, Theorem 1 , Chapter VI]. In the sequel, we omit ' $\left(\mathbb{R}^{n}\right)$ ' in the symbols of the function spaces.

3. The 1-norm approach. The following lemma is a variant of Hytönen's embedding result; see [5, Propositions 3.1 and 3.4]. Roughly speaking, it shows that if $q=t^{\prime}$, then one can reduce the index set, $\{0,1\}^{n}$, involved in the inequality (3.3) of $\left[5\right.$, Proposition 3.4], to the canonical basis of $\mathbb{R}^{n}$, i.e. $\left\{e_{i}\right\}_{i=1}^{n}$.

Lemma 3.1. Let $t \in[1,2]$ and $d \in \mathbb{N}$. Then there exists a constant $C$ such that for every $R>0$ and every $f \in L^{t}$ we have

$$
\int_{|x|>R}|\hat{f}(x)|^{t^{\prime}} \mathrm{d} x \leq C \sum_{i=1}^{n} \int_{0}^{1 / R}\left\|\Delta_{h e_{i}}^{d} f\right\|_{t}^{t^{\prime}} \frac{\mathrm{d} h}{h} .
$$


The proof follows the idea of the proofs of [5, Propositions 3.1 and 3.4].

Proof. Set $E(i, \rho, j):=\left\{x \in \mathbb{R}^{n}: 2^{j} \rho_{i} \leq\left|x_{i}\right|<2^{j+1} \rho_{i}\right\}$ for all $\rho \in(0, \infty)^{n}$, $i=1, \ldots, n$, and $j \in \mathbb{N}_{0}$. Let $E(i, \rho):=\bigcup_{j} E(i, \rho, j)(i=1, \ldots, n)$. Fix $R>0$. Note that $\{x:|x|>R\} \subset \bigcup_{i} E(i, \rho)$ for every $\rho \in(0, \infty)^{n}$ with $|\rho|_{\infty} \leq R / \sqrt{n}$. Moreover, since $\left|\left(1-e^{\mathbf{i} 2 \pi e_{i} \cdot x / 2 \sqrt{n} 2^{j} \rho_{i}}\right)\right| \geq C>0$ for every $x \in E(i, \rho, j)$, we get

$$
\begin{aligned}
\int_{E(i, \rho)}|\hat{f}(x)|^{t^{\prime}} \mathrm{d} x & \leq C \sum_{j} \int_{E(i, \rho, j)}\left|\left(1-e^{\mathbf{i} 2 \pi e_{i} \cdot x / 2 \sqrt{n} 2^{j} \rho_{i}}\right)^{d} \hat{f}(x)\right|^{t^{\prime}} \mathrm{d} x \\
& \leq C \sum_{j}\left\|\Delta_{e_{i} / 2 \sqrt{n} 2^{j} \rho_{i}}^{d} f\right\|_{t}^{t^{\prime}} .
\end{aligned}
$$

Therefore, taking the logarithmic average over $\rho_{i}$ and changing a variable $h=1 / 2 \sqrt{n} 2^{j} \rho_{i}$, we get

$$
\begin{aligned}
\int_{|x|>R}|\hat{f}(x)|^{t^{\prime}} \mathrm{d} x & \leq C \int_{R / 2 \sqrt{n} \bigcup_{i}} \int_{E(i, \rho)}^{R / \sqrt{n}}|\hat{f}(x)|^{t^{\prime}} \mathrm{d} x \frac{\mathrm{d} \rho_{i}}{\rho_{i}} \\
& \leq C \sum_{i} \sum_{j} \int_{R / 2 \sqrt{n}}^{R / \sqrt{n}}\left\|\Delta_{e_{i} / 2 \sqrt{n} 2^{j} \rho_{i}}^{d} f\right\|_{t}^{t^{\prime}} \frac{\mathrm{d} \rho_{i}}{\rho_{i}} \\
& \leq C \sum_{i} \sum_{j} \int_{1 / 2^{j+1} R}^{1 / 2^{j} R}\left\|\Delta_{h e_{i}}^{d} f\right\|_{t}^{t^{\prime}} \frac{\mathrm{d} h}{h} \\
& =C \sum_{i} \int_{0}^{1 / R}\left\|\Delta_{h e_{i}}^{d} f\right\|_{t}^{t^{\prime}} \frac{\mathrm{d} h}{h} .
\end{aligned}
$$

This completes the proof.

By a direct computation, Lemma 3.1 yields the following counterpart of $[5$, Lemma 4.1].

Lemma 3.2. Let $t \in[1,2], d \in \mathbb{N}$, and $\gamma \in(0,1]$. Suppose that $f \in L^{\infty}$ with $\operatorname{supp} f \subset\left\{x:|x| \leq 2^{\mu}\right\}(\mu \in \mathbb{Z})$ satisfies

$$
\left\|\Delta_{h e_{i}}^{d} f\right\|_{t} \leq 2^{\mu(n / t-\gamma d)} h^{\gamma d} \quad(i=1, \ldots, n, h>0) .
$$

Then there exists a constant $C$ such that

$$
\|\hat{f}\|_{t^{\prime}} \leq C 2^{\mu n / t} \text {, and }\left(\int_{|x|>R}|\hat{f}(x)|^{t^{\prime}} \mathrm{d} x\right)^{1 / t^{\prime}} \leq C 2^{\mu(n / t-\gamma d)} R^{-\gamma d}
$$

for every $R>0$. 
Lemma 3.2 motivates the following definition. For $0<p, q \leq \infty$ and $s>0$ we say that a function $m \in L^{\infty}$ satisfies the $B(s, p, q)$-condition, and write $m \in B(s, p, q)$ if there exists $\mathbb{N} \ni d \geq s$ such that

$$
\sup _{\substack{i=1, \ldots, n \\ \mu \in \mathbb{Z}}} \int_{0}^{\infty} h^{-s q}\left\|\Delta_{h e_{i}}^{d}\left[m\left(2^{\mu} \cdot\right) \phi\right]\right\|_{p}^{q} \frac{\mathrm{d} h}{h}<\infty, \quad B(s, p, q)
$$

with modification for $q=\infty$, i.e.

$$
\sup _{\substack{i=1, \ldots, n \\ \mu \in \mathbb{Z}, h>0}} h^{-s}\left\|\Delta_{h e_{i}}^{d}\left[m\left(2^{\mu} \cdot\right) \phi\right]\right\|_{p}<\infty . \quad B(s, p, \infty)
$$

It is equivalent to say that $m \in B(s, p, q)$ if

$$
\sup _{\mu \in \mathbb{Z}}\left\|m\left(2^{\mu} \cdot\right) \phi\right\|_{B_{p q}^{s}}<\infty \text {. }
$$

Indeed, combining [13, Theorems 2.5.12 and 2.5.13] with [13, Remark 3, p.113], for every $0<p, q \leq \infty, s>n(1 / \min (p, 1)-1)$, and $d>s$ we have

$$
B_{p, q}^{s}=\left\{f \in L^{p}: \max _{i=1, \ldots, n} \int_{0}^{\infty} h^{-s q}\left\|\Delta_{h e_{i}}^{d} f\right\|_{p}^{q} \frac{\mathrm{d} h}{h}<\infty\right\}
$$

and $\|\cdot\|_{p}+\max _{i=1, \ldots, n}\left(\int_{0}^{\infty} h^{-s q}\left\|\Delta_{h e_{i}}^{d}(\cdot)\right\|_{p}^{q} \frac{\mathrm{d} h}{h}\right)^{1 / q}$ is an equivalent quasi-norm in $B_{p, q}^{s}$ (modification if $q=\infty$ ).

Let $\lceil s\rceil$ denote the smallest integer at least $s$.

Lemma 3.3. Let $s>0,1 \leq p \leq \infty$, and $0<q \leq \infty$. Suppose that $m \in L^{\infty}$ satisfies the $B(s, p, q)$-condition with $d=\lceil s\rceil$.

Then, if $q<\infty$, we have

$$
\sup _{\substack{i=1, \ldots, n, \mu \in \mathbb{Z} \\ j=1, \ldots,\lceil s\rceil}} \int_{0}^{\infty} h^{-j \gamma q}\left\|\Delta_{h e_{i}}^{j}\left[m\left(2^{\mu} \cdot\right) \phi\right]\right\|_{p}^{q} \frac{\mathrm{d} h}{h}<\infty,
$$

for $\gamma:=s /\lceil s\rceil$ if $s \notin \mathbb{N}$, and for every $\gamma \in(0,1)$ if $s \in \mathbb{N}$.

If $q=\infty$, then

$$
\sup _{\substack{i=1, \ldots, \mu \in \mathbb{Z}, h>0 \\ j=0, \ldots,\lceil s\rceil}} h^{-\gamma j}\left\|\Delta_{h e_{i}}^{j}\left[m\left(2^{\mu} \cdot\right) \phi\right]\right\|_{p}<\infty
$$

for $\gamma=s /\lceil s\rceil$.

Proof. By (9) we have $m\left(2^{\mu} \cdot\right) \phi \in B_{p q}^{s}$ uniformly with respect to $\mu \in \mathbb{Z}$. Since $B_{p q}^{s} \subset W_{p}^{\lceil s\rceil-1}$ and

$$
h^{-j}\left\|\Delta_{h e_{i}}^{j} f\right\|_{p} \leq\left\|D^{j e_{i}} f\right\|_{p} \quad(i=1, \ldots, n, h>0, j=0, \ldots,\lceil s\rceil-1)
$$

for every $f \in W_{p}^{\lceil s\rceil-1}$, see e.g. [5, Corollary 2.2], we get

$$
\sup _{\substack{i=1, \ldots, n, h>0, \mu \in \mathbb{Z} \\ j=0, \ldots,\lceil s\rceil-1}} h^{-j}\left\|\Delta_{h e_{i}}^{j}\left[m\left(2^{\mu} \cdot\right) \phi\right]\right\|_{p}<\infty .
$$


The fact that we can replace $h^{-j}$ in (10) with $h^{-j \gamma}$ for every $\gamma \in(0,1)$ follows, for instance, from [5, Lemma 10.1]. This easily leads to the desired estimates.

Theorem 3.4. Let $m \in L^{\infty}$ satisfy the $B(s, t, \infty)$-condition for some $t \in[1,2]$ and $s>n / t$.

Then, for every multi-index $\beta \in \mathbb{N}_{0}^{n}$ with $|\beta|<s-n / t$, there exists a constant $C$ such that for every $R>0$ and $y \in \mathbb{R}^{n}$ with $0<2|y| \leq R$ we have

$$
\begin{aligned}
& \sum_{\mu \in \mathbb{Z}}\left(\int_{|x|>R}\left|D^{\beta} k_{\mu}(x)\right|^{1 / t^{\prime}} \mathrm{d} x\right)^{1 / t^{\prime}} \leq C R^{-n / t-|\beta|}, \quad \text { and } \\
& \sum_{\mu \in \mathbb{Z}}\left(\int_{|x|>R}\left|D^{\beta} k_{\mu}(x-y)-D^{\beta} k_{\mu}(x)\right|^{1 / t^{\prime}} \mathrm{d} x\right)^{1 / t^{\prime}} \\
& \quad \leq C R^{-n / t-|\beta|} \sigma\left(\frac{|y|}{R} ; s-\frac{n}{t}-|\beta|\right)
\end{aligned}
$$

where $\sigma(h ; \epsilon):=h^{\epsilon}$ if $\epsilon \in(0,1), \sigma(h ; \epsilon):=-h \log h$ if $\epsilon=1$, and $\sigma(h ; \epsilon):=h$ if $\epsilon>1(h \in(0,1))$.

In particular, $k \in K\left(t^{\prime}, s-n / t\right)$, and $k \in\left(D_{t^{\prime}}\right)$.

The proof follows the ideas provided in [5]. We give only the main supplementary observation which should be made, and leave the detailed verification for the reader.

Proof. By Lemma 3.3 and a change of variable, we get

$$
\begin{aligned}
\sup _{\substack{i=1, \ldots, n, h>0, \mu \in \mathbb{Z} \\
j=0, \ldots,\lceil s\rceil}} 2^{-\mu(n / t-\gamma j)} h^{-\gamma j}\left\|\Delta_{h e_{i}}^{j} m_{\mu}\right\|_{t} \\
=\sup _{\substack{i=1, \ldots, n, h>0, \mu \in \mathbb{Z} \\
j=0, \ldots,\lceil s\rceil}} h^{-\gamma j}\left\|\Delta_{h e_{i}}^{j}\left[m\left(2^{\mu} \cdot\right) \phi\right]\right\|_{t}<\infty,
\end{aligned}
$$

where $\gamma:=s /\lceil s\rceil$.

Therefore, since the set $\left\{j e_{i}: i=1, \ldots, n, j=0, \ldots, d\right\}$ is stable, $[5$, Lemma 11.1] (resp. [5, Lemma 11.2]) shows that the function $f(x):=x^{\beta} m_{\mu}(x)$ $\left(\right.$ resp. $\left.f(x):=x^{\beta} m_{\mu}(x)\left(1-e^{i 2 \pi s \cdot x}\right)\left(|s| \leq 2^{-\mu}\right)\right)\left(x \in \mathbb{R}^{n}, \mu \in \mathbb{Z}\right)$ satisfies the assumption of Lemma 3.2. By a straightforward computation, one can obtain the conclusion of [5, Lemma 11.3] with $q=t^{\prime}$ for all multi-indices $\beta$. Consequently, following the lines of the proofs of [5, Lemmas 11.4 and 11.5] we get also their conclusions for $q=t^{\prime}$ and all multi-indices $\beta$ with $|\beta|<s-n / t$. This yields the desired estimates.

Now, the second claim follows from the first one by a direct computation.

Remark 3.5. Note that the condition $\left(D_{r}\right)$ is monotonic with respect to $r \in$ $[1, \infty]$, i.e. $\left(D_{r_{2}}\right)$ implies $\left(D_{r_{1}}\right)$ if $1 \leq r_{1} \leq r_{2} \leq \infty$. In particular, if $k:=\widehat{m} \in$ 
$\left(D_{r}\right)(r \geq 1)$, then it satisfies the classical Hörmander condition, i.e. $\left(D_{1}\right)$, which shows that $m$ is a Fourier multiplier on all $L^{p}$ with $p \in(1, \infty)$.

The following corollary extends a result by Kurtz and Wheeden; see [8, Theorem 1].

Corollary 3.6. Suppose that $m \in B(s, t, \infty)$ for some $s>0$ and $t \in(1,2]$ such that $n / t<s \leq n$. If

(i) $\frac{n}{s}<p<\infty$ and $w \in A_{p s / n}$ or

(ii) $1<p<\left(\frac{n}{s}\right)^{\prime}$ and $w^{-1 /(p-1)} \in A_{p^{\prime} s / n}$,

then $m$ is a Fourier multiplier on $L_{w}^{p}$. Furthermore, if $s<n$, one may take $p=n / s$ in $(i)$, and $p=(n / s)^{\prime}$ in $(i i)$.

Proof. We make use of the generalisation of [8, Theorem 1] due to Rubio de Francia, Ruiz \& Torrea, see [11, Theorem 1.6, Part I], which in particular says that a singular integral operator of convolution type with kernel $k \in\left(D_{r}\right)$ $(1<r<\infty)$ is bounded on $L_{w}^{p}$ if $w \in A_{p / r^{\prime}}$ and $r^{\prime} \leq p<\infty$ or if $w \in A_{p}^{r^{\prime}}$ and $1<p \leq r$. Note that by a kernel in [11] the authors mean a function in $L_{l o c}^{1}\left(\mathbb{R}^{n} \backslash\{0\}\right)$. However, the conclusion of [11, Theorem 1.6, Part I] still holds in our a bit more general case of $k=\widehat{m}$ with $m \in L^{\infty}$ and $k \in\left(D_{r}\right)$. Indeed, according to the above definition, one can apply [11, Theorem 1.6, Part I] to $\sum_{\mu=-\nu}^{\nu} k_{\mu}(\nu \in \mathbb{N})$ and then the standard approximation and density arguments.

To apply this result, first observe that the condition $B(s, t, \infty)$ is monotonic with respect to $t$, i.e. if $1 \leq t_{1} \leq t_{2} \leq 2$, then $B\left(s, t_{2}, \infty\right)$ yields $B\left(s, t_{1}, \infty\right)$. Indeed, since $\Delta_{y}^{j}\left[m\left(2^{\mu} \cdot\right) \phi\right]=\sum_{i \leq j}\left(\begin{array}{c}j \\ i\end{array}\right)(-1)^{i} \tau_{i y}\left[m\left(2^{\mu} \cdot\right) \phi\right]\left(y \in \mathbb{R}^{n}\right)$, the claim follows immediately from Hölder's inequality.

Now, if $w \in A_{p s / n}$, then there exists $r \in(1, \infty)$ such that $n / s<r^{\prime}<$ $\min (p, t)$ with $w \in A_{p / r^{\prime}}$. By the above observation and Theorem 3.4 , we get $k \in\left(D_{r}\right)$, and consequently $(i)$ holds. By the standard duality argument one can show $(i i)$.

Since for every $w \in A_{r}(r \geq 1)$ there exists $\epsilon>1$ such that $w^{\epsilon} \in A_{r}$, and $m$ is a multiplier on $L^{p}$ for every $p>1$, see Remark 3.5, the last statement can be obtained by an interpolation argument with a change of measure. This completes the proof.

We conclude with further results on multipliers on weighted Hardy spaces which extend slightly the results due to Strömberg and Torchinsky in [12]. We start with preliminary observations.

First, note that if $m \in M(t, l)(t \in[1,2], l>0)$, then $m \in B(l, t, \infty)$. Indeed, this follows, for instance, from [5, Lemma 4.2] by taking as the stable set $\mathcal{I}$ in its formulation the set $\left\{j e_{i}: i, \ldots, n, j=0, \ldots,\lceil l\rceil\right\}$, and noting that in this case $|\alpha|_{1}=|\alpha|_{\infty}$ for all $\alpha \in \mathcal{I}$.

We point out that the multiplier results of Strömberg and Torchinsky [12, Theorems 4, 5, and 6, Chapter XI] are formulated in the terms of the condition $M(t, l)$, though, their proofs are based on the behaviour of $\widehat{m}$ reflecting the fact that $m \in M(t, l)$. In other words, [12, Theorems 4,5 , and 6 , Chapter XI] follow 
from corresponding convolution variants for a kernel $\widehat{m}$ which are implicitly provided therein on the way in accordance to the fact that $m \in M(t, l)$ implies $\widehat{m} \in K\left(t^{\prime}, l-n / t\right)$; see [12, Lemma 1 , Chapter XI].

Therefore, combining Theorem 3.4 with the above observation, we get the following extension of [12, Theorems 4, 5, and 6, Chapter XI]. We refer the reader, for instance, to [12, Chapter I] for the definition of the notions involved in its formulation, i.e. the Muckenhoupt $A_{p}$-condition, the reverse Hölder $R H_{r^{-}}$ condition, and the doubling $D_{b}$-condition. Moreover, following [12], we set $r_{w}:=\sup \left\{r \geq 1: w \in R H_{r}\right\}$ for the critical index of the weight $w \in A_{\infty}$ for the reverse Hölder condition.

Theorem 3.7. Let $m \in L^{\infty}$ and $r>1$. Suppose that $m \in B(s, t, \infty)$ for some $t \in[1,2]$ and $s>n / t$. Then the following assertions hold.

(i) For every $r \leq p \leq \infty$ and $w \in A_{r}$ with $n \min \left(\frac{r}{p}, \frac{1}{p^{\prime}}-\frac{1}{r_{w} p}\right)<s, m$ is a Fourier multiplier on $L_{w}^{p}$.

(ii) For every $0<p<r$ and $w \in A_{r} \cap D_{\theta n}$ with $1 \leq \theta \leq r$ and

$$
s> \begin{cases}\frac{n(\theta-1)(s-p)}{p(r-1)}+n \max \left(\frac{1}{t}, \frac{1}{p^{\prime}}+\frac{1}{r_{w} p}\right) & \text { if } 1<p<r, \\ n \max \left(\frac{\theta}{p}-\frac{1}{t^{\prime}}, \frac{\theta}{p}-\frac{r_{w}-1}{r_{w} p}\right) & \text { if } 0<p \leq 1,\end{cases}
$$

$m$ is a Fourier multiplier on $H_{w}^{p}$.

(iii) For every $p \in(0,1]$ with $s>n\left(1 / p-1 / t^{\prime}\right), m$ is a Fourier multiplier on $H^{p}$.

Remark 3.8. a) Combining Lemma 3.1, (9), and Hölder's inequality we immediately get that for every $s \geq 0, t \in[1,2]$, and $t^{\prime} \leq q \leq \infty$ the Fourier transform maps $B_{t, q}^{s}$ in $K_{t^{\prime}, q}^{s}$ continuously; see (6) for the definition of $K_{t^{\prime}, q}^{s}$. Note that Lemma 3.2 is a special variant of these embeddings for $q=\infty$.

b) In [1], Baernstein and Sawyer proved that if $p \in(0,1)$, and either $s=$ $n(1 / p-1 / 2)$ and $0<q \leq p$ or $s>n(1 / p-1 / 2)$ and $q \in(0, \infty]$, then the condition

$$
\sup _{\delta>0}\|m(\delta \cdot) \phi\|_{B_{2, q}^{s}}<\infty,
$$

or by (9), equivalently $m \in B(s, 2, q)$, implies that $m$ is a Fourier multiplier on $H^{p}$; see [1, Corollary 1]. This result is sharp in the sense that $B_{2, q}^{s}$ cannot be replaced by any larger Besov space $B_{2, t}^{r}$. Recently related conditions were systematically studied by Kolomoitsev; see [6,7] and Remark $4.5 \mathrm{~b}$ ). Moreover, note that Theorem 3.7 ( iii) reproduces the BaernsteinSawyer result for $t=2$ and $q=\infty$, and recall that $B_{2, q_{1}}^{s} \subset B_{2, q_{2}}^{s}$ if $q_{1}<q_{2}$. It is not clear for the author if one can sharpen the statements (ii) and ( iii) of Theorem 3.7 if $m \in B(s, 2, q)$ with $0<q \leq p$ and $s=n(1 / p-1 / 2)$.

c) The statements $(i)$ and $(i i)$ of Theorem 3.7 correspond directly to $[12$, Theorems 5 and 6, Chapter XI]. The last one (iii) is an immediate consequence of ( $i i)$ (by taking a constant weight), and corresponds to [12, Corollary, p. 164]. Note that, by a standard interpolation argument for 
the $H^{p}$-scale, $($ iii $)$ implies that $m$ is a multiplier on $H^{p}$ for all $p>1$, and recall that $H^{p}=L^{p}$ if $p>1$. However, it should be underlined that the proof of $(i)$ and $(i i)$ is based on the fact that $m$ is a multiplier on $L^{p}$ spaces for appropriate values $p>1$, which follows, for instance, from the fact that $\widehat{m}$ satisfies the classical Hörmander condition, see Remark 3.5 (cf. also the proof of [12, Theorem 4]).

4. The $\infty$-norm approach. In this section, we adapt the $\infty$-norm approach established by Hytönen in [5] to provide an extension of Kolomoitsev's results; see Remark $4.5 \mathrm{~b}$ ). The condition below allows one to control the $\infty$-norm of dyadic parts $m_{\mu}$ of multiplier functions $m$, as well as the $\infty$ - and 1-norm of multi-indices of their difference operators.

Theorem 4.1. Let $m \in L^{\infty}$. Suppose that for some $\rho>0, t \in[1,2], d \in \mathbb{N}$, and $\gamma \in(0,1]$ with $\gamma d>1 / t$ we have

$$
\sup _{\mu \in \mathbb{Z}, h \in(0, \infty)^{n}}\left\|m\left(2^{\mu} \cdot\right) \phi\right\|_{\infty}^{\rho}\left\|h^{-\gamma \alpha} \partial_{h}^{\alpha}\left[m\left(2^{\mu} \cdot\right) \phi\right]\right\|_{t}<\infty
$$

for all multi-indices $\alpha$ with $|\alpha|_{\infty} \leq d$.

Then $m$ is a Fourier multiplier on $H^{p}$ for every $p \in(0,2]$ such that

$$
\frac{1}{p}-\frac{1}{2}<\frac{1}{1+\rho}\left(\frac{\gamma d}{n}+\frac{1}{2}-\frac{1}{t n}\right) .
$$

The following corollary follows straightforward from [5, Lemma 10.4].

Corollary 4.2. Let $m \in L^{\infty}$. Suppose that for some $\rho>0, t \in[1,2]$, and $d \in \mathbb{N}$ we have

$$
\sup _{\mu \in \mathbb{Z}, h \in(0, \infty)^{n}}\left\|m\left(2^{\mu} \cdot\right) \phi\right\|_{\infty}^{\rho}\left\|h^{-\alpha} \partial_{h}^{\alpha}\left[m\left(2^{\mu} \cdot\right) \phi\right]\right\|_{t}<\infty
$$

for all multi-indices $\alpha$ with $|\alpha|_{\infty} \leq d$ and $|\alpha| \leq l$, where $\lfloor n / t\rfloor+1 \leq l \leq n d$ if $d \geq 2$ or $d=1$ and $t \neq 1$, and $l=n$ if $d=t=1$.

Then the conclusion of Theorem 4.1 holds with $\gamma=l / n d$.

Remark 4.3. In the case of $\inf _{\mu \in \mathbb{Z}}\left\|m_{\mu}\right\|_{\infty}>0$, the condition (11) is equivalent to Hytönen's one from [5, Corollary 9.6], i.e. $\sup _{\mu \in \mathbb{Z}, h \in(0, \infty)^{n}} \| h^{-\gamma \alpha} \partial_{h}^{\alpha}$ $\left[m\left(2^{\mu} \cdot\right) \phi\right] \|_{t}<\infty$, which gives the conclusion of Theorem 4.1 with $\rho=0$.

Corollary 4.4. Let $m \in L^{\infty}$ with $|m(x)| \leq C \frac{1}{(1+|x|)^{a}}\left(x \in \mathbb{R}^{n}\right)$ for some constants $a>0$ and $C>0$. If

$$
\sup _{\mu \in \mathbb{Z}, h \in(0, \infty)^{n}} \min \left(1,2^{-\mu b}\right)\left\|h^{-\alpha} \partial_{h}^{\alpha}\left[m\left(2^{\mu} \cdot\right) \phi\right]\right\|_{t}<\infty
$$

for some $b \geq 0$ and all $\alpha \in\{0,1\}^{n}$ with $|\alpha| \leq l:=\lfloor n / t\rfloor+1$, where $t \in(1,2]$, then the conclusion of Theorem 4.1 holds with $\rho=b / a, d=1$, and $\gamma=l / n$.

Proof of Theorem 4.1. The proof follows a standard interpolation argument. Set

$$
m(z, \cdot):=\sum_{\mu \in \mathbb{Z}}\left\|m\left(2^{\mu} \cdot\right) \phi\right\|_{\infty}^{\rho-(1+\rho) z} m \phi\left(2^{-\mu} \cdot\right) \quad(0 \leq \Re z \leq 1)
$$


First, note that $m(1+\mathbf{i} y, \cdot)$ are Fourier multipliers on $H^{2}=L^{2}$ uniformly with respect to $y \in \mathbb{R}$, and

$$
m\left(\mathbf{i} y, 2^{\mu} \cdot\right) \phi=\sum_{|\nu-\mu| \leq 1}\left\|m\left(2^{\mu} \cdot\right) \phi\right\|_{\infty}^{\rho-(1+\rho) i y} m\left(2^{\mu} \cdot\right) \phi\left(2^{\mu-\nu} \cdot\right) \phi .
$$

By [5, Lemma 10.1], there exists a constant $C$ such that

$$
\left\|\partial_{h}^{\beta} \phi\left(2^{\mu-\nu} \cdot\right)\right\|_{\infty} \leq C h^{\gamma \beta} 2^{|\beta|}\left\|D^{\beta} \phi\right\|_{\infty}
$$

for every $h \in(0, \infty)^{n},|\nu-\mu| \leq 1$, and every $\beta \in \mathbb{N}_{0}^{n}$ with $|\beta|_{\infty} \leq d$. Therefore, combining Leibniz' formula

$$
\partial_{h}^{\alpha}\left[m\left(2^{\mu} \cdot\right) \phi\left(2^{\mu-\nu} \cdot\right) \phi\right]=\sum_{\beta \leq \alpha}\left(\begin{array}{c}
\alpha \\
\beta
\end{array}\right) \partial_{h}^{\alpha-\beta}\left[m\left(2^{\mu} \cdot\right) \phi\right] \tau_{(\alpha-\beta) h}\left[\partial_{h}^{\beta} \phi\left(2^{\mu-\nu} \cdot\right)\right]
$$

with (11), we get

$$
\left\|\partial_{h}^{\alpha}\left[m\left(\mathbf{i} y, 2^{\mu} \cdot\right) \phi\right]\right\|_{t} \leq C h^{\gamma \alpha} \quad\left(\mu \in \mathbb{Z}, h \in(0, \infty)^{n}\right)
$$

for all $|\alpha|_{\infty} \leq d$. Consequently, by [5, Corollary 9.6], $m(\mathbf{i} y, \cdot), y \in \mathbb{R}$, are Fourier multipliers on $H^{q}$ for every $q \in(0,1)$ with $\gamma d>1 / t+n(1 / q-1)$ uniformly with respect to $y$.

Finally, note that for every $p \in(0,1)$ satisfying $(12)$, there exists $q \in(0,1)$ with $\gamma d>1 / t+n(1 / q-1)$ such that for $\theta:=(1+\rho)^{-1}$ we have $\theta / q+(1+\theta) / 2=$ $1 / p$. Thus, by the complex interpolation for the $H^{p}$-scale, see [2, Theorem 3.4], we get that $m_{\theta}=m$ is a Fourier multiplier on $H^{p}$. This completes the proof.

Remark 4.5. a) Let $\alpha \in \mathbb{N}_{0}^{n}$ and $m \in L^{\infty}$ be such that $D^{\alpha} m \in L_{l o c}^{1}$. By [5, Corollary 2.2], for all $q \in[1, \infty]$ we have that

$$
\left\|\partial_{h}^{\alpha} m\right\|_{q} \leq\left|h^{\alpha}\right|\left\|D^{\alpha} m\right\|_{q} \quad\left(h \in(0, \infty)^{n}\right) .
$$

Therefore, the assumption of Theorem 4.1 can be checked in the terms of the classical Hörmander-type conditions $M(l, t)$ introduced in Section 2. In particular, it gives the proof of Theorem 1.1 in Section 1.

Moreover, recall that the function $m_{\epsilon, \delta}(\xi):=\psi(\xi) e^{\mathbf{i}|\xi|^{\epsilon}}|\xi|^{-\delta}(\xi \in$ $\left.\mathbb{R}^{n}\right)$, where $\epsilon, \delta>0$ and $\psi \in \mathcal{C}^{\infty}$ with $\psi(\xi)=0$ for $|\xi| \leq 1$ and $\psi(\xi)=0$ for $|\xi| \geq 2$, is a Fourier multiplier on $H^{p}$ if and only if $\delta \geq n(1 / p-1 / 2) \epsilon$; see e.g. [10, Proposition 5.1]. By direct computation, one can show that for an appropriate choice of $\epsilon$ and $\delta$ the function $m=m_{\epsilon, \delta}$ satisfies the condition (3) for some $b>0$ but not $\sup _{\mu \in \mathbb{Z}, \alpha \in \mathcal{I}_{l, d}} \| 2^{\mu\left(|\alpha|_{1}-n / t\right)} \chi_{S_{\mu}}$ $D^{\alpha} m \|_{t}<\infty$.

b) It may be also interesting to compare Corollary 4.2 with Kolomoitsev's results from [7] following the remark stated below Theorem A in Section 1. For the convenience of the reader we restate here [7, Theorems 3 and 4]. We replace the classical Besov norm $\|\cdot\|_{B_{p, q}^{s}}$ occurring in [7] with the equivalent one used in the previous section; see (9). 
Theorem B (Kolomoitsev, [7, Theorems 3 and 4]). Let $m \in L^{\infty}$.

(i) If

$$
\sup _{\mu \in \mathbb{Z}, h>0}\left\|m\left(2^{\mu} \cdot\right) \phi\right\|_{\infty}^{\rho}\left\|h^{-s} \Delta_{h e_{i}}^{\lfloor s\rfloor+1}\left[m\left(2^{\mu} \cdot\right) \phi\right]\right\|_{t}<\infty
$$

for some $\rho>0$ and $s>n / \min (t, 2)$ with $t \in(0, \infty]$, then $m$ is a Fourier multiplier on $H^{p}$ for all $p \in[1,2]$ with $1 / p-1 / 2 \leq s /(1+\rho) n$.

(ii) If

$$
\sup _{\mu \in \mathbb{Z}, h>0}\left\|m\left(2^{\mu} \cdot\right) \phi\right\|_{\infty}^{\rho}\left\|h^{-s} \Delta_{h e_{i}}^{\lfloor s\rfloor+1}\left[m\left(2^{\mu} \cdot\right) \phi\right]\right\|_{\infty}<\infty
$$

for some $\rho>0$ and $s>0$, then $m$ is a Fourier multiplier on $H^{p}$ for all $p \in(1,2]$ with $1 / p-1 / 2 \leq s /(1+\rho) n$.

In particular, note that if $s \in \mathbb{N}$, then

$$
C^{s} \subsetneq\left\{f \in L^{\infty}: D^{s e_{i}} f \in L^{\infty}, i=1, \ldots, n\right\} \subsetneq B_{\infty \infty}^{s} \subsetneq C^{s-1}
$$

continuously. Here, the derivatives $D^{s e_{i}}$ are considered in the sense of distributions. The second inclusion follows from the estimates $\left\|\Delta_{h e_{i}}^{s} f\right\|_{\infty} \leq h^{s}\left\|D^{s e_{i}} f\right\|_{\infty}$ $(h>0)$; see e.g. [5, Lemma 2.1].

Open Access. This article is distributed under the terms of the Creative Commons Attribution 4.0 International License (http://creativecommons.org/licenses/ by/4.0/), which permits unrestricted use, distribution, and reproduction in any medium, provided you give appropriate credit to the original author(s) and the source, provide a link to the Creative Commons license, and indicate if changes were made.

\section{References}

[1] A. Baernstein II And E. T. SAwyer, Embedding and multiplier theorems for $H^{p}\left(\mathbb{R}^{n}\right)$, Mem. Amer. Math. Soc. 53 (1985), iv+82.

[2] A. Calderón and A. Torchinsky, Parabolic maximal functions associated with a distribution. II, Adv. Math. 24 (1977), 101-171.

[3] L. De-Michele and I. R. Inglis, $L^{p}$ estimates for strongly singular integrals on spaces of homogeneous type, J. Funct. Anal. 39 (1980), 1-15.

[4] L. HÖRMANDER, Estimates for translation invariant operators in $L^{p}$ spaces, Acta Math. 104 (1960), 93-140.

[5] T. Hүтӧnen, Fourier embeddings and Mihlin-type multiplier theorems, Math. Nachr. 274/275 (2004), 74-103.

[6] Yu. S. Kolomoitsev, Generalization of one sufficient condition for Fourier multipliers, Ukrainian Math. J. 64 (2013), 1562-1571.

[7] Yu. S. Kolomoitsev, Multiplicative sufficient conditions for Fourier multipliers, Izv. Ross. Akad. Nauk Ser. Mat. 78 (2014), 145-166.

[8] D. S. Kurtz And R. L. Wheeden, Results on weighted norm inequalities for multipliers, Trans. Amer. Math. Soc. 255 (1979), 343-362. 
[9] A. Miyachi, On some Fourier multipliers for $H^{p}\left(\mathbb{R}^{n}\right)$, J. Fac. Sci. Univ. Tokyo Sect. IA Math. 27 (1980), 157-179.

[10] A. Mryachi, On some singular Fourier multipliers, J. Fac. Sci. Univ. Tokyo Sect. IA Math. 28 (1981), 267-315.

[11] J. L. Rubio de Francia, F. J. Ruiz, and J. L. Torrea, Calderón-Zygmund theory for operator-valued kernels, Adv. Math. 62 (1986), 7-48.

[12] J.-O. Strömberg And A. Torchinsky, Weighted Hardy spaces, Lecture Notes in Mathematics, vol. 1381, Springer, Berlin, 1989.

[13] H. Triebel, Theory of function spaces, Monographs in Mathematics, vol. 78, Birkhäuser, Basel, 1983.

Sebastian KRóL

Institut für Analysis,

Fachrichtung Mathematik,

TU Dresden, 01062 Dresden,

Germany

and

Faculty of Mathematics and Computer Science,

Nicolaus Copernicus University,

ul. Chopina 12/18,

87-100 Toruń, Poland

e-mail: sebastian.krol@mat.umk.pl

Received: 26 May 2015

Revised: 11 December 2015 